(C) [2007] IEEE. Reprinted, with permission, from [Marc G Carmichael, Dikai Liu, Kenneth J Waldron, Investigation of reducing fatigue and musculoskeletal disorder with passive actuators, Intelligent Robots and Systems (IROS), 2010 IEEE/RSJ International Conference on, 18-22 Oct. 2010]. This material is posted here with permission of the IEEE. Such permission of the IEEE does not in any way imply IEEE endorsement of any of the University of Technology, Sydney's products or services. Internal or personal use of this material is permitted. However, permission to reprint/republish this material for advertising or promotional purposes or for creating new collective works for resale or redistribution must be obtained from the IEEE by writing to pubs-permissions@ieee.org. By choosing to view this document, you agree to all provisions of the copyright laws protecting it 


\title{
Investigation of Reducing Fatigue and Musculoskeletal Disorder with Passive Actuators
}

\author{
Marc G Carmichael, Dikai Liu, Kenneth J Waldron \\ Centre for Autonomous Systems \\ University of Technology, Sydney \\ Broadway 2007, NSW. Australia \\ marc.g.carmichael@eng.uts.edu.au, dkliu@eng.uts.edu.au, \\ kenneth.waldron@uts.edu.au
}

\begin{abstract}
Robotic systems such as exoskeletons can be effectively used in the reduction of fatigue and musculoskeletal disorders (MSD) associated with physical tasks, but robots which work in physical contact with humans pose problems with user safety. A novel approach to developing intrinsically safe robots is to use passive actuators which have the advantage of being safer, ensuring stability, high force/weight ratios and lower power consumption. It is however not clear how effective an exoskeleton utilizing passive actuators would be in reducing fatigue and the risk of MSD. This paper analyzes the benefit of using such a system with results from dynamic simulations and an experiment using a specially designed mechanism used for evaluation. Results indicate that fatigue and effort could be reduced if robot impedance is minimized. Experiments also highlighted issues of implementing such a system into practice.
\end{abstract}

\section{INTRODUCTION}

Musculoskeletal disorders (MSDs) refer to conditions that involve the nerves, tendons, muscles, and supporting structures of the body [1]. In the United States it is estimated that MSDs account for nearly 130 million health care encounters annually. Conservative estimates have direct compensation costs of workplace MSDs around \$20 billion, and including indirect cost estimates raises this as high as $\$ 45$ to $\$ 54$ billion dollars annually [2].

Sources of MSD include a mismatch of the task's physical demand and biological compatibility, repeated and prolonged performing of tasks, and physical exertion which exceeds the tolerance of the musculoskeletal system which is a function of force duration, posture and motion [3]. Risk of MSD can therefore be reduced by using robots to augment a person performing physical labor, either to reduce the magnitude or the duration of the load exerted on the person during the task. Robots have been used to great effect in the reduction of MSDs in industry, for example Intelligent Assist Devices (IADs) [4]. Another solution are robotic exoskeletons [5], [6], [7], [8] where the robot is worn and operates in parallel with the operator to achieve a task. Advantages of exoskeletons over other robotic solutions are that they are intuitive to use, and are capable of being operated without requiring large and costly infrastructure. Robotic exoskeletons are therefore suitable for assisting with physical tasks in human domains.

Before robots can work in physical contact with humans the aspect of human safety needs to be addressed. This is of particular importance with exoskeletons where the operator is physically constrained to the robot. Industrial manipulators achieve safety by isolating robots and humans. When this is not possible, other methods for achieving safety in physical human-robot interaction (pHRI) are required. Controlling robot force instead of position control can attempt to keep interaction forces between robot and human at safe levels [9]. The controller may also limit the robot's maximum velocity, acceleration, static force, and dynamic power to safe levels for human proximity as suggested by the latest revisions to standard ISO 10218 [10]. Another approach to safe pHRI is to design robots with intrinsic mechanical safety by such means as reducing robot inertia, so in case of collision the transfer of energy to the human is reduced to acceptable safe levels. An example is the DLR series of robots [9] which combine a light weight structure with torque sensing and high performance actuation to achieve intrinsically safe manipulators capable of payloads equal their own weight. Adding mechanical compliance is another method of reducing the apparent inertia of the robot by decoupling the actuator inertia from the end effector [11]. These methods typically increase safety at the expense of performance, since reducing link weight can introduce deflection in the structure, and compliance can result in slower response times, larger settling times and increased oscillations [12].

A novel approach to developing intrinsically safe robots is to use passive actuators. We define passive actuators as mechanical devices which can be actively controlled, but unlike active actuators, can only absorb energy and cannot introduce energy into a system (except for energy initially stored in the device). An example of such passive actuators are magnetorheological dampers and magnetic particle brakes. Since passive actuators can only absorb energy then the risk of transmitting enough energy to a human to cause injury is reduced. Mechanically passive robots ensure stability at all frequencies, and in the case of malfunction they do not produce high velocities resulting in potentially dangerous levels of kinetic energy [13]. Mechanical, electrical or control failure could still occur using passive actuators, but because this risk also exists with active actuators then in comparison we consider them a safer (but possibly not as beneficial) alternative. Passive devices can also have higher force/weight ratios and lower power consumption compared to similar 
performance active actuators. An obvious disadvantage is that a purely passive robot cannot move against external forces unless assisted by some other means. This external energy can be supplied by a human operator working in parallel with the robot by way of pHRI.

For an exoskeleton system, replacing its active actuators with passive ones limits the robot to only providing isometric forces or dynamic forces against the direction of movement, only capable of negative work, as opposed to positive work which is performed while providing force in the same direction as displacement. The human is required to provide all the positive work needed to achieve the physical task. An example of such a system is the wearable chair [14] which provides isometric forces allowing the device to act as a chair, but when standing or walking the operator provides all of the required power. Another system is the Cobot [15] which uses an active means of mechanically constraining relative joint velocities in a robot. This allows the implementation of virtual walls in the work space to aid the user, for example to help guide large loads on a Cobot trolley [12]. Although not in exoskeleton form, such a device still requires the user to provide the positive energy needed to complete the task, while aiding the user through actively controlled passive means.

What is not clear is how effective an exoskeleton system utilizing this passive approach would be in reducing fatigue and MSD. Physical tasks involve both positive and negative work, but a passive exoskeleton system can only assist with the latter. Furthermore, friction and inertia of the robot will increase the effort required from the operator when positive work is performed. This paper analyzes the benefit of utilizing a theoretical passive robotic exoskeleton to reduce fatigue and MSD during physical tasks. In section II a dynamic human/exoskeleton model is used to simulate two industrial tasks. A fatigue model is used to estimate the passive robot's effect on the operators fatigue. Section III describes an experiment in which a passive brake was utilized to assist persons in performing a simple lifting task. Conclusions are detailed in section IV.

\section{SIMULATION}

Two common industrial scenarios were chosen to simulate a person at risk of fatigue and MSD. Scenario 1 is a person repeatedly lowering mass $m$ from a high to lower position, much like a person unloading goods from a truck. The mass is lifted at above shoulder height, brought in towards their chest, and then placed in a lowered position in front of the human. The human then repeats the same movements in reverse without carrying any external mass. The movement cycle takes 2 seconds during which the mass is lowered a total of $0.71 \mathrm{~m}$ and the torso rotates 90 degrees in the transverse plane.

Scenario 2 is a person performing a sandblasting operation with their upper arm approximately vertically downwards and elbow bent at 90 degrees. A force is exerted at the hand along the forearm to replicate the reaction force of the sandblasting nozzle. The hand is cycled in a rectangular pattern $0.3 \mathrm{~m} \times 0.1 \mathrm{~m}$ on a flat plane $0.3 \mathrm{~m}$ in front of the user, mimicking the sandblasting of a flat wall. The movement cycle takes 4 seconds to complete. These two scenarios were chosen as it is expected both would benefit from the passive exoskeleton system, with trajectories based on observations of persons performing similar tasks.

\section{A. Simulation Setup}

The simulation was implemented in Matlab using the Robotics Toolbox [16]. A simplified 3DOF dynamic model of a human was created which included torso rotation in the transverse plane, shoulder flexion/extension, and elbow flexion/extension. Other arm articulations were not included since they are minimally used in the two scenarios. Length, mass, and inertial properties of the model's body segments were based on published anthropometric data and equations [17], [18], [19]. The simulation consists of a single human arm, with the external load halved to what would be experienced in both arms.

The human joint torques required for the motions of scenarios 1 and 2 were calculated using inverse dynamics based on the dynamic equation (1) where $q, \dot{q}, \ddot{q}$ are joint coordinate, velocity and acceleration vectors, $\mathbf{M}$ is the joint space inertia matrix, $\mathbf{C}$ represents Coriolis and centripetal effects, $\mathbf{G}$ is the gravity loading, $\mathbf{J}$ is the robot Jacobian and $F_{R}$ is a vector of external forces in task space. Combining joint torques and displacement, the positive and negative work was calculated. The positive work performed by the human is the combination of the work required to move the external load, exoskeleton, and the user's arm. The only negative work performed by the human is the energy required to move their own arm, since the exoskeleton can support itself and the external load. In comparison, when no exoskeleton is being utilized the amount of positive work required is less since no exoskeleton needs to be moved, however in negative work the user is required to support the entire external load themselves with no assistance.

$$
\tau=\mathbf{M}(q) \ddot{q}+\mathbf{C}(q, \dot{q}) \dot{q}+\mathbf{G}(q)+\mathbf{J}(q)^{T} F_{R}
$$

A fatigue model from [20] estimated the fatigue in the shoulder and elbow, but was modified to separate flexion and extension motions of the joints since isolated muscles are used. Initially the strength capacity of each motion $\left(T_{c}\right)$ is set to its maximum isometric strength $\left(T_{\max }\right)$ published in [21]. During the simulation when there exists a motion torque, the fatigue model reduces the motion's strength capacity using (2). When there is no motion torque then the capacity is recovered using (3). $k$ and $R$ are fatigue and recovery parameters and $T$ is the current motion torque. Fatigue is defined as when any motion strength capacity is below the strength needed to perform the task.

$$
\begin{gathered}
\dot{T}_{c}=-\left(k T_{c} T\right) / T_{\max } \\
\dot{T}_{c}=R\left(T_{\max }-T_{c}\right)
\end{gathered}
$$




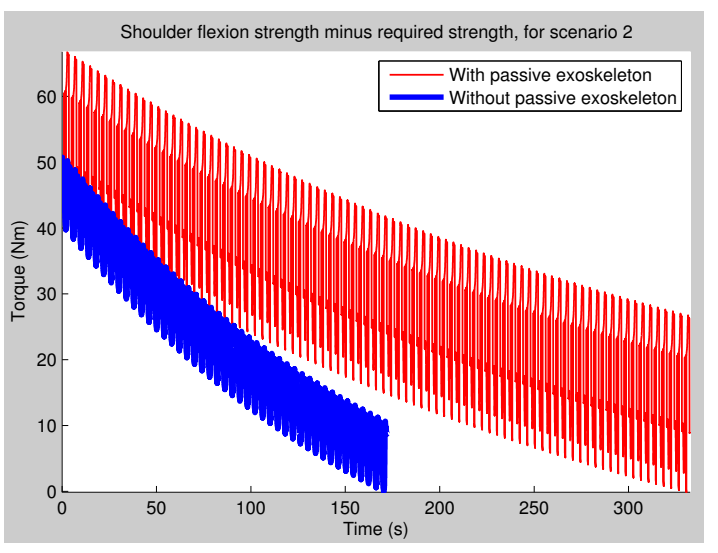

Fig. 1. Difference between shoulder flexion strength and required strength in scenario 2. Fatigue is reached when difference is zero

Exoskeleton mass and inertia increase the effort required when performing positive work. To analyze this in our simulation we define a parameter $\beta$ by which the mass and inertia of the model is scaled. For $\beta=1$ the exoskeleton has zero mass/inertia, $\beta=2$ the exoskeleton has the same mass/inertia as the human arm, and $\beta=3$ it has double. The simulation is repeated with $\beta$ ranging from 1 to 3 . For scenario 1 , the simulation is repeated as the lifted mass $m$ is varied from 1 to $10 \mathrm{~kg}$, since $20 \mathrm{~kg}$ ( $10 \mathrm{~kg}$ per arm) is an appropriate limit for safe manual lifting [22]. For scenario 2 the reaction force $F_{r}$ is varied from $10 \mathrm{~N}$ to $100 \mathrm{~N}$, with $50 \mathrm{~N}$ (per arm) being appropriate for this type of task [23].

\section{B. Simulation results}

In both simulated scenarios the use of the passive exoskeleton was able to significantly reduce operator fatigue. In scenario 1 with $\beta=2$ and $m=10 \mathrm{~kg}$ without exoskeleton aid the person first reached fatigue in shoulder flexion after 40 seconds. With exoskeleton aid this time was increased to 82 seconds. In scenario 2 with $\beta=2$ and $F_{r}=50 \mathrm{~N}$ without exoskeleton aid, fatigue was first reached in shoulder flexion after 170 seconds. With exoskeleton aid this increased to 330 seconds, as seen in Fig. 1 with curves showing the difference between the available joint strength and the strength required to perform the task, with fatigue reached when this difference reaches zero.

The work required from the human was reduced by utilizing the passive exoskeleton in both scenarios. For scenario 1 , the largest percentage of energy saved by using the exoskeleton was $63 \%$ when $m=10 \mathrm{~kg}$ and $\beta=1$, and for scenario 2 was $41 \%$ when $F_{r}=100 \mathrm{~N}$ and $\beta=1$. With large exoskeleton inertia and low external loads, the human energy expenditure increased indicating the system has no positive benefit. The larger the external load the less $\beta$ had an effect on the energy saved. Fig. 2 shows the percentage of human energy saved in scenario 2 by utilizing the exoskeleton for various external loads and exoskeleton mass/inertia. The black line indicates where the surface crosses $0 \%$. Results from all simulations are shown in Table I.

With the fatigue model used, if the external loads are

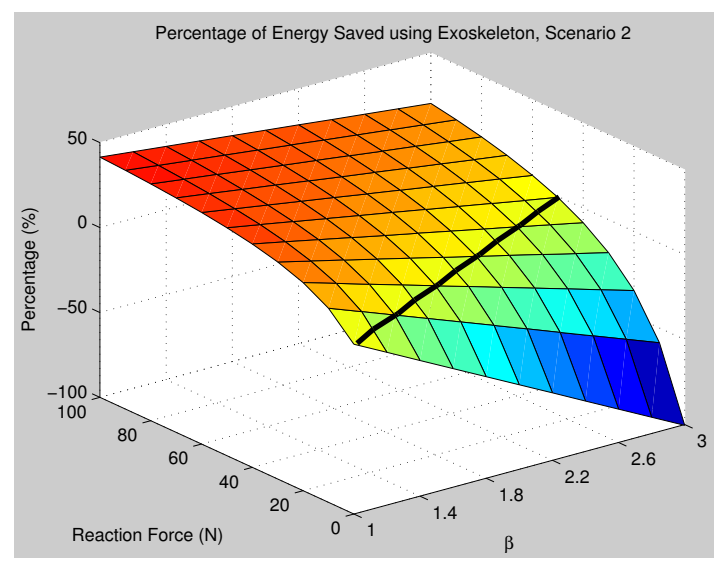

Fig. 2. Reduction of human energy by using exoskeleton in scenario 2

TABLE I

SiMULATION RESULTS

\begin{tabular}{c|c|c|c|c}
\multicolumn{5}{c}{ Scenario 1 } \\
\hline$\beta$ & $\begin{array}{c}m \\
(\mathrm{~kg})\end{array}$ & $\begin{array}{c}\text { Time till fatigue } \\
\text { without exoskeleton }\end{array}$ & $\begin{array}{c}\text { Time till fatigue } \\
\text { with exoskeleton }\end{array}$ & $\begin{array}{c}\text { Percentage of } \\
\text { energy saved }\end{array}$ \\
\hline \hline 1 & 8 & $182.7 \mathrm{~s}$ & $457.8 \mathrm{~s}$ & $59.8 \%$ \\
1 & 10 & $96.3 \mathrm{~s}$ & $214.8 \mathrm{~s}$ & $63.2 \%$ \\
2 & 6 & $152.6 \mathrm{~s}$ & $333.3 \mathrm{~s}$ & $38.5 \%$ \\
2 & 8 & $82.3 \mathrm{~s}$ & $170.6 \mathrm{~s}$ & $46.5 \%$ \\
2 & 10 & $40.1 \mathrm{~s}$ & $82.3 \mathrm{~s}$ & $52.0 \%$ \\
3 & 6 & $70.2 \mathrm{~s}$ & $38.5 \mathrm{~s}$ & $22.0 \%$ \\
3 & 8 & $34.1 \mathrm{~s}$ & $66.2 \mathrm{~s}$ & $33.2 \%$ \\
3 & 10 & $10.0 \mathrm{~s}$ & $18.0 \mathrm{~s}$ & $40.7 \%$ \\
\hline
\end{tabular}

Scenario 2

\begin{tabular}{c|c|c|c|c}
\hline$\beta$ & $\begin{array}{c}F_{r} \\
(\mathrm{~N})\end{array}$ & $\begin{array}{c}\text { Time till fatigue } \\
\text { without exoskeleton }\end{array}$ & $\begin{array}{c}\text { Time till fatigue } \\
\text { with exoskeleton }\end{array}$ & $\begin{array}{c}\text { Percentage of } \\
\text { energy saved }\end{array}$ \\
\hline \hline 1 & 50 & $266.8 \mathrm{~s}$ & $502.9 \mathrm{~s}$ & $34.3 \%$ \\
1 & 80 & $122.5 \mathrm{~s}$ & $260.9 \mathrm{~s}$ & $39.4 \%$ \\
1 & 100 & $78.4 \mathrm{~s}$ & $172.7 \mathrm{~s}$ & $41.4 \%$ \\
2 & 50 & $170.3 \mathrm{~s}$ & $330.6 \mathrm{~s}$ & $16.8 \%$ \\
2 & 80 & $86.4 \mathrm{~s}$ & $178.3 \mathrm{~s}$ & $26.8 \%$ \\
2 & 100 & $54.3 \mathrm{~s}$ & $122.0 \mathrm{~s}$ & $30.9 \%$ \\
3 & 80 & $57.9 \mathrm{~s}$ & $118.1 \mathrm{~s}$ & $14.3 \%$ \\
3 & 100 & $37.8 \mathrm{~s}$ & $78.0 \mathrm{~s}$ & $20.3 \%$ \\
\hline
\end{tabular}

small enough, the joint torque capacity will fatigue to a steady-state level above the maximum required joint strength, implying that the task could be performed indefinitely. This is because other fatigue factors such as posture discomfort and metabolic constraints are not accounted for. In our simulations with very small external loads results did show that fatigue would never be reached, however it is realized that this is not correct.

\section{EXPERIMENT}

To test the passive actuator concept in reality a simple test rig was designed in which a passive actuator assisted the user while performing a manual lifting task. A magnetic particle brake was selected for actuation for its good force rendering 
ability and low time constant [24]. Although the test platform was highly structured and resembled an IAD more than an exoskeleton, it allows the passive actuator concept to be experimented without having to invest in the development of a complete wearable robot. A control strategy was formulated which adjusts the braking torque so that when lowering the mass a set percentage of the load force is removed from the user. Experiments were performed in which the forces from the human, brake and the external load were measured for various levels of robot assistance.

\section{A. Experiment Setup}

Fig. 3 shows the test rig. A bearing-mounted hollow aluminium shaft fitted with a handle allows a participant to apply force to turn the shaft. One end of the shaft is connected to a magnetic particle brake via a planetary gearbox. The other end is fitted with a pulley from which a mass is suspended, and a potentiometer to measure shaft position. When the user applies a force at the handle the shaft turns, lifting or lowering the suspended mass. A multi-axis load cell (ATI Nano25) measures human torque input at the handle. Two strain gauges located on the hollow shaft allow the torque produced by the brake and the suspended mass to be calculated. Combining force measurements with shaft displacement the human work input, brake assisting work, and combined output work used to lift and lower the external mass was calculated. The magnetic particle brake (Merobel FAS2) is capable of producing up to $0.2 \mathrm{Nm}$ braking torque or 62.8 Watts of braking power. This type of brake is attractive as its torque is almost linearly proportional to the electric current applied, allowing for open-loop control. Dimensions of the brake are $45 \times 45 \times 47.5 \mathrm{~mm}$, it weighs $200 \mathrm{~g}$

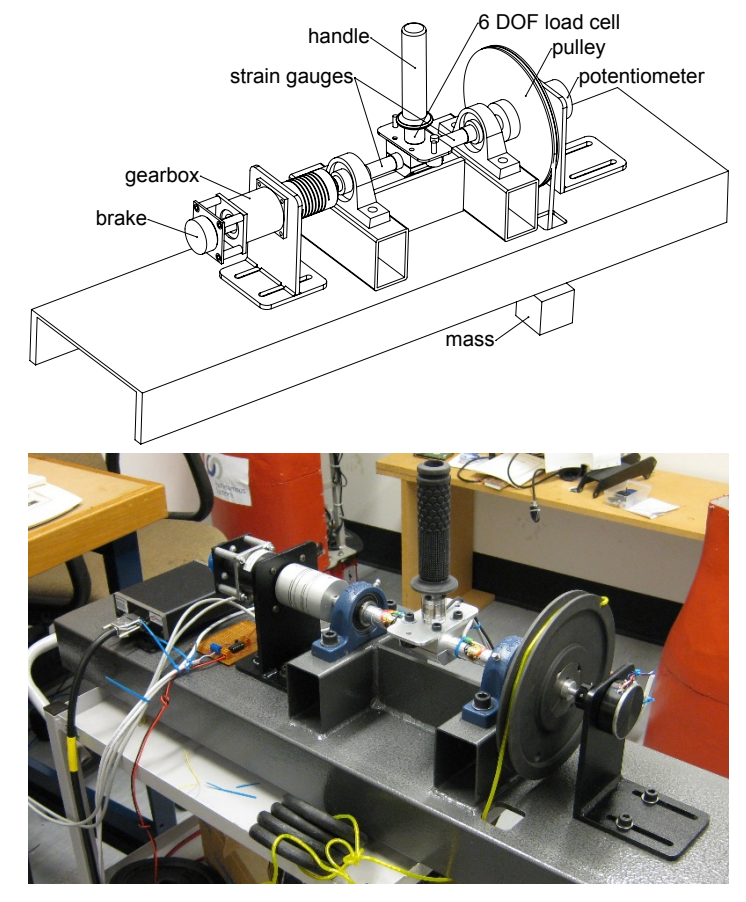

Fig. 3. Passive brake test rig and consumes a maximum of 0.5 Watts of electrical energy. Although this device uses electrical energy during operation, it is still passive since none of this energy is positively transferred to the mechanical system.

\section{B. Control Algorithm}

To formulate a control function for the magnetic particle brake we define the following parameters:

$T_{H} \quad$ Torque from human input $(\mathrm{Nm})$

$T_{M} \quad$ Torque from suspended mass $(\mathrm{Nm})$

$T_{B 0} \quad$ Magnitude of brake torque $(\mathrm{Nm})$

$\omega \quad$ Shaft angular velocity ( $\mathrm{rad} / \mathrm{s})$

$\alpha \quad$ Shaft angular acceleration $\left(\mathrm{rad} / \mathrm{s}^{2}\right)$

$J_{0} \quad$ Shaft moment of inertia $\left(\mathrm{kg}-\mathrm{m}^{2}\right)$

Taking Newton's second law about the shaft's axis of rotation we obtain (4), where $\operatorname{SIGN}(\omega)$ is the direction of shaft rotation. $T_{B 0}$ is always positive and proportional to the current applied to the brake.

$$
T_{H}-T_{M}-\operatorname{SIGN}(\omega) \times T_{B 0}=J_{0} \alpha
$$

The aim of the control scheme is to reduce the force at the handle by a set factor, while still applying a force to the user proportional to the external load, but reduced. By defining $\lambda$ as a factor of how much of the external torque is portrayed to the user we obtain:

$$
\begin{gathered}
T_{H}=\lambda T_{M} \\
T_{B 0}=S I G N(\omega) \times\left[T_{M}(\lambda-1)-J_{0} \alpha\right]
\end{gathered}
$$

The control system calculates the required braking torque from (6). If the result is negative then this indicates that a torque from the brake in the same direction as shaft rotation is required in order to maintain (5). Since this is not possible with such a passive device, the braking torque is set to zero.

During the experiments participants were asked to repeatedly lift and lower the suspended mass, following a desired position indicator shown on a computer screen which oscillated sinusoidally at $0.05 \mathrm{~Hz}$ with magnitude of 60 degrees. Each experiment lasted 300 seconds and was repeated with $\lambda$ values of $0,0.25,0.5,0.75$ and 1 . When $\lambda=1$ the brake is never utilized and the entire weight of the mass is supported by the participant at all times. When $\lambda=0$ the brake ideally supports all the external load during lowering of the mass.

\section{Experimental Results}

Initially the behavior of the system was noticeably jittery when the shaft was either stationary or during transition between lowering and lifting phases, see Fig. 4. To overcome this a small bias was added to the shaft velocity measurement before calculating $T_{B 0}$. This reduced jittering when the shaft was stationary, but was still noticeable at transition between lifting and lowering phases with $\lambda$ values of 0 and 0.25 . It is believed this behavior is due to the cable suspending the mass acting as a spring, causing the brake to 
TABLE II

EXPERIMENT RESULTS

\begin{tabular}{c|c|c|c|c|c|c}
\hline$\lambda$ & $\begin{array}{c}\text { Human work } \\
\text { during lifting }(\mathbf{J})\end{array}$ & $\begin{array}{c}\text { Human work } \\
\text { during lowering }(\mathbf{J})\end{array}$ & $\begin{array}{c}\text { Total human } \\
\text { work }(\mathbf{J})\end{array}$ & $\begin{array}{c}\text { External load } \\
\text { mechanical energy }(\mathbf{J})\end{array}$ & $\begin{array}{c}\text { Brake electrical } \\
\text { consumption }(\mathbf{J})\end{array}$ & $\begin{array}{c}\text { Estimated metabolic } \\
\text { cost }(\mathbf{J})\end{array}$ \\
\hline \hline 1.00 & 3576 & -2868 & 6446 & 6332 & 0.00 & 32104 \\
0.75 & 3600 & -2531 & 6144 & 6405 & 4.19 & 31291 \\
0.50 & 3569 & -1740 & 5321 & 6317 & 17.42 & 28807 \\
0.25 & 3586 & -855.3 & 4435 & 6285 & 40.40 & 26373 \\
0.00 & 3801 & -68.38 & 3867 & 6367 & 72.16 & 25537 \\
\hline
\end{tabular}

be quickly engaged and disengaged in response to vibration. When $\lambda$ was set to $0.5,0.75$ or 1 this effect was barely evident. The acceleration term used in (6) calculated by taking the double derivative of shaft position contained a lot of noise which also contributed to the jittering effect, and was consequently ignored when calculating $T_{B 0}$. This was considered acceptable since accelerations were small during the experiments.

A summary of the results collected from one of the participants is shown in Table II, with results being similar among all participants. The total human work is the sum of the positive and absolute negative work. Using the brake the total human work was reduced from $6446 \mathrm{~J}(\lambda=1)$ to 3867J $(\lambda=0)$, a reduction of $40 \%$ while requiring only $72.16 \mathrm{~J}$ of electrical energy. With the brake deactivated the human work was greater than the mechanical work required to lift the mass due to friction in the device, however it was around $2 \%$ greater indicating that friction losses were not significant. As expected the negative work performed by the participant reduced as $\lambda$ was changed from 1 to 0 , while the positive work did not significantly change since the brake is not utilized during lifting. A small increase in positive work was noticed when $\lambda=0$ due to increased effort required to combat the previously mentioned jittering. The average error between the desired and actual shaft position during the experiments did not appear to relate to the amount of assistance being provided by the brake, indicating that in the experiments the use of the passive brake to assist the user had little effect on their ability to control the lifting/lowering of the mass (except for the jittering effect).

It is worthwhile noting that the efficiency of human

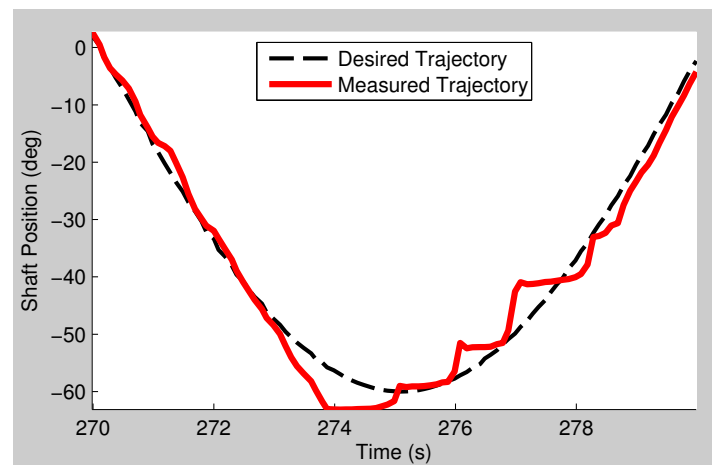

Fig. 4. Jittery behavior at $\lambda=0$ muscle converting chemical energy into mechanical energy (mechanochemical efficiency) is not the same when performing concentric and eccentric muscle movement, with published efficiencies of $15 \%$ and $34.7 \%$ for positive and negative work respectively [25]. Interestingly, other research has shown concentric and eccentric muscle contraction to have similar decreases in muscle strength over time due to fatigue [26], [27]. Applying these efficiencies to the experimental results reduces the $40 \%$ mechanical energy saving to only a $20 \%$ metabolic saving, but it is not known how this is reflected in the user's fatigue.

Apart from the inherent safety, other benefits of the passive approach is the weight and power consumption of the system. To highlight this we compare the experimental results to theoretical results achieved if an active actuator was used instead. As a theoretical substitute for the brake the Maxon RE 30 range of DC motors are chosen since they have a similar power rating of 60 Watts. This motor weighs $238 \mathrm{~g}$ and has $87 \%$ maximum efficiency, in comparison to the FAS2 brake which weights less yet has a braking efficiency of $12560 \%$. The ratio of human mechanical energy saved to actuator mass for the brake is $12.9 \mathrm{~J} / \mathrm{gram}$. The active motor could potentially provide all the mechanical energy required, resulting in a ratio of $26.6 \mathrm{~J} / \mathrm{gram}$. Comparing these values the passive approach is vastly more efficient in providing the mechanical work saving to the user for an equivalent electrical consumption, however it has only $48 \%$ of the human work benefit per unit mass. It is clear that the brake is a better solution if low power consumption is a key requirement. Since power supplies such as batteries can be a large proportion of a robotic system's weight, taking this into account then the passive approach could be more favorable for a light weight system. If not taking this into account then the active approach is more suited.

\section{CONCLUSIONS AND FUTURE WORK}

Both the simulation and experiment showed a significant reduction in the amount of work required in performing certain physical tasks when being augmented with passive actuators. Benefits increase in tasks which involve higher levels of negative work, for example unloading goods off a truck. Tasks which don't involve predominately negative work can still benefit from this approach with increases in task duration before the person reaches a fatigued state, as shown in simulation. The experiment validated the simulation in showing that a saving in work can be achieved during a 
task. Issues highlighted by the implementation were undesirable behavior at the transition between positive and negative work, and mechanical losses in the system requiring the user to provide more effort when performing positive work.

Observing the effect $\beta$ had on the system showed increasing exoskeleton mass reduces the benefit to the user, since when performing positive work the user is required to move the exoskeleton in addition to their own arm and any external loads. If the mass is great enough then the exoskeleton will have a net negative effect, increasing the amount of energy required from the user when performing physical tasks. Large exoskeleton mass also puts extra load on other parts of the body (for example, the users legs). Mass is therefore a critical design constraint in passive exoskeletons for reducing fatigue and MSD.

It has been shown that a robotic system such as an exoskeleton utilizing passive actuators could be used to reduce the magnitude and duration of loads experienced by persons performing physical tasks, and such use could reduce fatigue and risk of MSD. Drawbacks from the passive approach are that the user cannot be assisted during phases of positive work, however for certain applications where the weight and power consumption are critical design factors (such as the case for mobile exoskeletons and similar) utilizing passive actuators could give the system desirable properties such as being light weight, low power consumption, and having the inherent user safety associated with passive systems. It is possible that utilizing passive actuators makes some applications feasible, where using an active approach they are not.

Future work will look at the implementation of a fatigue model based on the fatigue of individual muscles rather than the net fatigue around the joints of the model. This will result in a better understanding of the fatigue, with more confidence in the results. Simulations could also be based on actual recorded tasks using sensors to measure motions and external forces. Development of a passive exoskeleton will allow the simulation results to be compared with experimental results obtained in a similar scenario. Furthermore the benefit of the passive system could be examined by measuring the reduction in EMG signals or oxygen consumption of the user.

\section{REFERENCES}

[1] U.S. Department of Health and Human Services, Musculoskeletal Disorders and Workplace Factors, Cincinnati, OH, July 1997, no. 97B141.

[2] National Research Council, Panel on Musculoskeletal Disorders and the Workplace, and Commission on Behavioral and Social Sciences and Education, Musculoskeletal Disorders and the Workplace : Low Back and Upper Extremities. Washington, DC: National Academies Press, 2001.

[3] S. Kumar, "Theories of musculoskeletal injury causation," $E r$ gonomics, vol. 44, no. 1, pp. $17-47,2001$.

[4] K. L. Tan, "Intelligent assist device: design and development," in NCTT 2003 Proceedings. 4th National Conference on Telecommunication Technology, January 2003, pp. 198- 202.

[5] M. Bergamasco, B. Allotta, L. Bosio, L. Ferretti, G. Parrini, G. Prisco, F. Salsedo, and G. Sartini, "An arm exoskeleton system for teleoperation and virtual environments applications," Robotics and Automation, 1994. Proceedings., 1994 IEEE International Conference on, vol. 2, pp. 1449-1454, May 1994.
[6] M. Brown, N. Tsagarakis, and D. Caldwell, "Exoskeletons for human force augmentation," Industrial Robot: An International Journal, vol. 30, no. 6, pp. 592-602, 2003.

[7] J. Perry, J. Rosen, and S. Burns, "Upper-limb powered exoskeleton design," Mechatronics, IEEE/ASME Transactions on, vol. 12, no. 4, pp. 408-417, Aug 2007.

[8] E. Rocon, A. Ruiz, J. Pons, J. Belda-Lois, and J. Sanchez-Lacuesta, "Rehabilitation robotics: a wearable exo-skeleton for tremor assessment and suppression," in Robotics and Automation, 2005. ICRA 2005. Proceedings of the 2005 IEEE International Conference on, April 2005, pp. 2271-2276.

[9] G. Hirzinger, A. Albu-Schaffer, M. Hahnle, I. Schaefer, and N. Sporer, "On a new generation of torque controlled light-weight robots," in Proceedings 2001 ICRA. IEEE International Conference on Robotics and Automation, vol. 4. Inst. of Robotics \& Mechatronics, German Aerosp. Center, Wessling, Germany;, 2001, pp. 3356- 3363.

[10] ISO 10218-1:2006, Robots for industrial environments - Safety requirements - Part 1: Robot. ISO, Geneva, Switzerland, 2006.

[11] G. A. Pratt, M. M. Williamson, P. Dillworth, J. Pratt, K. Ull, and A. Wright, "Stiffness isn't everything," in Proceedings of ISER, 1995, pp. 253-262.

[12] A. Bicchi, M. Peshkin, and J. E. Colgate, "Safety for physical human-robot interaction," in Handbook of Robotics, B. Siciliano and O. Khatib, Eds. Berlin: Springer, 2008, pp. 1335-1348.

[13] M. Peshkin, J. E. Colgate, and C. Moore, "Passive robots and haptic displays based on nonholonomic elements," in Proceedings of the 1996 IEEE International Conference on Robotics and Automation, April 1996.

[14] T. Mitsuda, M. Wakabayashi, and S. Kawamura, "Development of wearable chair using pneumatic passive elements," Journal of Robotics and Mechatronics, vol. 16, no. 3, pp. 256-263, 2004.

[15] M. A. Peshkin, J. E. Colgate, W. Wannasuphoprasit, C. A. Moore, R. B. Gillespie, and P. Akella, "Cobot architecture," Robotics and Automation, IEEE Transactions on, vol. 17, no. 4, pp. 377-390, 2001.

[16] P. Corke, "A robotics toolbox for MATLAB," IEEE Robotics and Automation Magazine, vol. 3, no. 1, pp. 24-32, mar 1996.

[17] D. A. Winter, Biomechanics and motor control of human movement, 2nd ed. Ontario, Canada: A Wiley-Interscience publication, 1990.

[18] C. C. Gordon, T. Churchill, C. E. Clauser, B. Bradtmiller, J. T. McConville, I. Tebbetts, and R. A. Walker., "1988 anthropometric survey of u.s. army personnel: Summary statistics interim report," United States Army Natick Research, Development and Engineering Centre, Yellow Springs, Ohio, Tech. Rep., March 1989.

[19] R. F. Chandler, C. E. Clauser, J. T. McConville, H. M. Reynolds, and J. W. Young, "Investigation of inertial properties of the human body," U.S Department of Transportation, Tech. Rep., March 1975.

[20] L. Ma, D. Chablat, F. Bennis, W. Zhang, and F. Guillaume, "A new muscle fatigue and recovery model and its ergonomics application in human simulation," CoRR, 2009.

[21] S. Kumar, Ed., Muscle Strength. CRC Press, 2004.

[22] N. O. Health and A. Safety Commission, Manual handling : National standard for manual handling (NOHSC:1001(1990)) / National code of practice for manual handling (NOHSC:2005(1990)) / National Occupational Health and Safety Commission. Australian Govt. Pub. Service, Canberra, 1991.

[23] N. Kirchner, G. Paul, and D. Liu, "Bridge maintenance robotic arm: mechanical technique to reduce the nozzle force of a sandblasting rig," Journal of Wuhan University of Technology, vol. 28, pp. 12-18, 2006.

[24] F. Conti and O. Khatib, "A new actuation approach for haptic interface design," The International Journal of Robotics Research, vol. 28, no. 6, pp. 834-848, 2009.

[25] T. W. Ryschon, M. D. Fowler, R. E. Wysong, A. R. Anthony, and R. S. Balaban, "Efficiency of human skeletal muscle in vivo : comparison of isometric, concentric, and eccentric muscle action," Journal of Applied Physiology, vol. 83, no. 3, pp. 867-874, September 1997.

[26] M. J. Mullaney and M. P. McHugh, "Concentric and eccentric muscle fatigue of the shoulder rotators," International Journal of Sports Medicine, vol. 27, no. 9, pp. 725-729, 2006.

[27] Y. Kawakami, H. Kanehisa, S. Ikegawa, and T. Fukunaga, "Concentric and eccentric muscle strength before, during and after fatigue in 13 year-old boys," European Journal of Applied Physiology and Occupational Physiology, vol. 67, no. 2, pp. 121-124, August 1993. 